Article

\title{
One-Step Synthesis of High Pure Tris(8-hydroxyquinoline)aluminum for Optics and Photonics
}

\author{
Roman Avetisov, Ksenya Kazmina, Artem Barkanov (D), Marina Zykova, Andrew Khomyakov, Alexander Pytchenko \\ and Igor Avetissov*(D)
}

check for updates

Citation: Avetisov, R.; Kazmina, K.; Barkanov, A.; Zykova, M.; Khomyakov, A.; Pytchenko, A.; Avetissov, I. One-Step Synthesis of High Pure Tris(8-hydroxyquinoline) aluminum for Optics and Photonics. Materials 2022, 15, 734. https:// doi.org/10.3390/ma15030734

Academic Editor: Massimo Cocchi

Received: 29 November 2021

Accepted: 17 January 2022

Published: 19 January 2022

Publisher's Note: MDPI stays neutral with regard to jurisdictional claims in published maps and institutional affiliations.

Copyright: (C) 2022 by the authors. Licensee MDPI, Basel, Switzerland. This article is an open access article distributed under the terms and conditions of the Creative Commons Attribution (CC BY) license (https:// creativecommons.org/licenses/by/ $4.0 /)$.
Department of Chemistry and Technology of Crystals, Mendeleev University of Chemical Technology, 125047 Moscow, Russia; armoled@mail.ru (R.A.); kazmina.k.v@muctr.ru (K.K.); barkanov.a.d@muctr.ru (A.B.); zykova_mp@inbox.ru (M.Z.); homa-ifh@yandex.ru (A.K.); pytchenkoaa@mail.ru (A.P.)

* Correspondence: igor_avetisov@mail.ru

\begin{abstract}
A simple method of synthesis of high pure tris(8-hydroxyquinoline)aluminum (Alq ${ }_{3}$ ) from commercial available $5 \mathrm{~N} \mathrm{Al}_{2} \mathrm{O}_{3}$ and 8-hydroxyquinolinol has been developed. One-step exchange chemical reaction has been conducted under controlled 8-hydrixyquinoline vapor at a temperature of $190-240{ }^{\circ} \mathrm{C}$ with water removal by phosphorus anhydride. According to analysis of inductively coupled plasma mass-spectrometry, the chemical purity of synthesized $\mathrm{Alq}_{3}$ was $99.998 \mathrm{wt} \%$. Photoluminescence of the synthesized $\mathrm{Alq}_{3}$ has been measured and slightly differed from those of $\mathrm{Alq}_{3}$ obtained by traditional organic synthesis.
\end{abstract}

Keywords: tris(8-hydroxyquinoline)aluminum; pure substance; inductively coupled plasma mass spectrometry

\section{Introduction}

In the last two decades, a large effort has been directed towards development of wearable/implantable electronics based on organic semiconducting materials. The most recent developments include environmental monitoring, implantable medical devices, on-skin sensors, and disposable plastic electronics such as e-tickets, RFID tags, plastic cards, etc. [1-14]. These devices require plentiful and low-cost materials and production technologies supporting their dynamic development. Organic semiconducting material technologies are interesting for their fine-tuning of characteristics due to the large variability of chemical formulas at high productivity and low production costs.

To date OLED technologies have become widespread in various fields of techniques: perfect TV displays, energy efficient lighting devices, IR sensors and displays for medical application, etc. Tris(8-hydroxyquinoline)aluminum $\left(\mathrm{Alq}_{3}\right)$ was the first OLED emission material [15] and till now it has a wide application both as an emitting material and an electron transport material for cheap commercial devices [1].

Conceptually, OLED devices are electronic semiconductor structures [6], and, as in the case of inorganic semiconductors, organic materials that are used in multilayer OLED structures must meet the requirements for semiconductors. In particular, the chemical purity of organic semiconductors must be as high as inorganic ones. The successful development of technologies for inorganic semiconductors and devices based on them began more than 70 years ago, just when the chemical purity of $99.999 \mathrm{wt} \%(5 \mathrm{~N})$ became generally available (Figure 1). To date, modern inorganic semiconductors are characterized by a purity of $99.99999 \mathrm{wt} \%$ (7N) for GaAs technologies [16] to $99.999999999 \mathrm{wt} \%$ (11N) for silicon technologies [17]. 


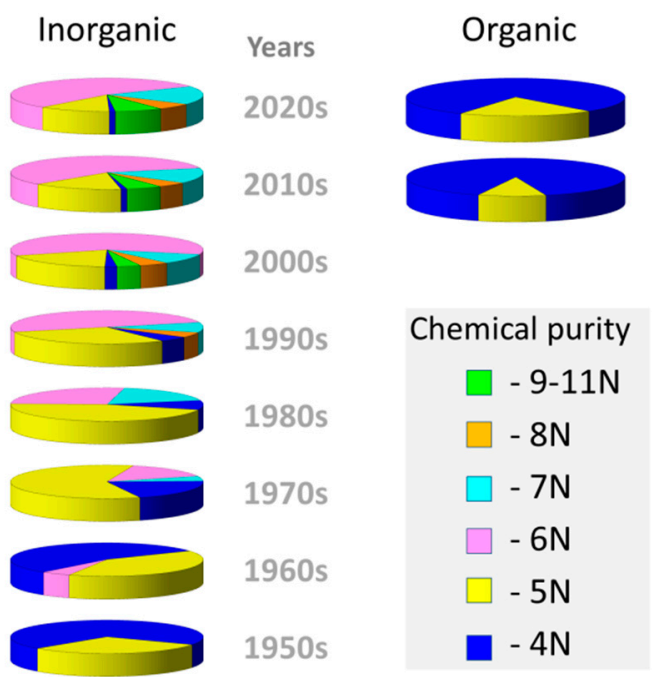

Figure 1. Production dynamic (rel.\%) of inorganic (left column) and organic (right column) semiconducting materials having different chemical purity $(\mathrm{xN})$.

Organic semiconducting materials (including phosphors for OLED technology) are generally produced by conducting of a chemical reaction in a complex liquid media. This technology needs pure solvents, organic precursors and multistep purification procedures.

For instance, the production of high pure $\mathrm{Alq}_{3}(99.995 \mathrm{wt} \%$ ) still remains a comparatively expensive: the price for sublimated $\mathrm{Alq}_{3} 99.9-99.999 \mathrm{wt} \%$ is $60-100 \mathrm{kEuro} / \mathrm{kg}[18,19]$. The standard procedure includes the reaction of complex formation of aluminum salt (chloride, nitrate) with 8-hydroxyquinolinol in isopropanol solution with further sedimentation by ammonia hydroxide, multiply washing by isopropanol, and finally vacuum sublimation [20]. This procedure needs high pure initial reagents and special extra-pure chemical equipment.

In the presented research, we developed a simple one-step method of synthesis of tris(8-hydroxyquinolinate)aluminum from cheap commercial preparations with $5 \mathrm{~N}$ and $6 \mathrm{~N}$ chemical purity. The synthesized $\mathrm{Alq}_{3}$ preparation was formed on the surface of $\mathrm{Al}_{2} \mathrm{O}_{3}$ grains and the synthesized heterophase preparation could be a source of the chemical pure $\mathrm{Alq}_{3}$ for OLED technology.

\section{Materials and Methods}

\subsection{Impurity Determination by ICP-MS}

To analyze chemical purity of initial and final preparations, we used inductively coupled plasma mass spectrometry with preliminary transfer of the solid sample to the liquid phase by dissolving them in high-purity nitric $\left(\mathrm{HNO}_{3}\right)$ acid (7N7), purified by a Berghof BSB-939-IR surface distillation system (Berghof GmbH, Eningen, Germany) or high-purity sulfuric $\left(\mathrm{H}_{2} \mathrm{SO}_{4}\right)$ acid (8N Ultrapur, Sigma-Aldrich Chemie $\mathrm{GmbH}$, Taufkirchen, Germany) in a SPEEDWAVE-FOUR microwave decomposition system (Berghof GmbH, Eningen, Germany) equipped with DAP-100 PTFE autoclaves (Berghof GmbH, Eningen, Germany). We used extra pure water (AquaMax-Ultra 370 Series, Young Lin Instruments Co., Ltd., Anyang, South Korea) with a specific resistance of $18 \mathrm{M} \Omega \cdot \mathrm{cm}$ for dilution.

Analytical measurements were carried out on a NexION 300D inductively coupled plasma mass spectrometer (ICP-MS) (PerkinElmer Inc., Waltham, MA, USA). The TotalQuant method for determination of 65 chemical elements' concentrations was used [21] with the operating parameters presented in Table 1. 
Table 1. The operating mode of the NexION 300D instrument for conducting impurity analysis of samples.

\begin{tabular}{lc}
\hline Nebulizer type & Concentric (Meinhard), PFA \\
Spray chamber & Scott double-pass chamber, PFA \\
Argon flow rate, L/min & 0.96 \\
through the nebulizer & 15 \\
plasma-forming & 1.2 \\
auxiliary & 1450 \\
Generator power, $W$ & 4.6 \\
Collision gas (He) flow rate, L/min & 8 \\
Number of scan cycles & \\
\hline
\end{tabular}

\subsection{Initial Preparations}

Powder $\mathrm{Al}_{2} \mathrm{O}_{3}$ purchased from Prima Ltd. (Korolev, Russia) was used as an Al-source for $\mathrm{Alq}_{3}$ synthesis. The above preparation is usually used for sapphire crystal growth for laser applications. According to the ICP-MS analysis (Figure 2), it was as pure as $99.998 \mathrm{wt} \%$ (65 elements detected). A preliminary heat-treated $(870 \mathrm{~K}) \mathrm{Al}_{2} \mathrm{O}_{3}$ powder preparation was used in a synthesis procedure.

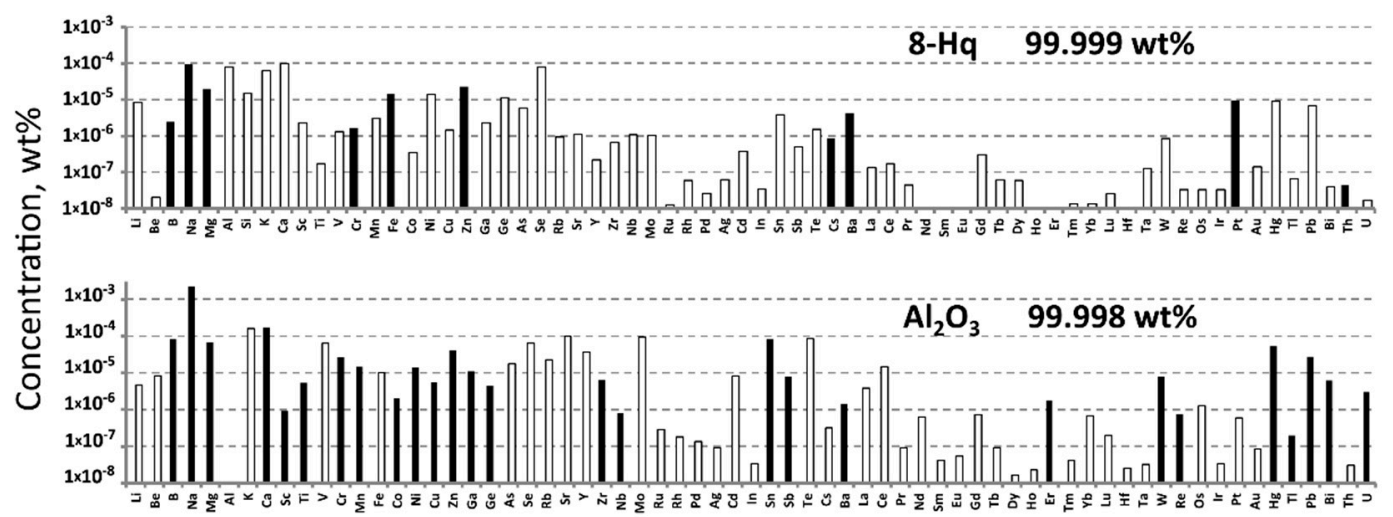

Figure 2. Impurity concentrations determined by ICP-MS in the initial preparations. Here and after, the empty (white) bars indicate the limits of determination (LD) of ICP-MS analysis. The concentrations of the non-presented elements were less $10^{-8} \mathrm{wt} \%$.

8-hydroxyquionolinol (8-Hq) purchased from Komponent Reaktive Ltd. (Moscow, Russia) was additionally purified by vacuum sublimation to the chemical purity of $99.999 \mathrm{wt} \%$ determined by 65 elements (Figure 2). As-sublimated 8-Hq was used directly in a synthesis procedure.

\subsection{SEM and Optical Microscopy Analysis}

To analyze the preparation morphology, we used optical and electron microscopies. We used a Stereo Discovery V.12 binocular microscope (CarlZeiss, Oberkochen, Germany) with white and UV lighting.

SEM images of powder preparations were obtained using a VEGA-3 LMU scanning electron microscope (TESCAN ORSAY HOLDING, Brno-Kohoutovice, Czech Republic) in secondary electron (SE) mode with $5 \mathrm{kV}$ accelerating voltage.

\subsection{Spectral Parameter Measurements}

All of the luminescence measurements were carried out at room temperature. We used a Fluorolog FL3-22 spectrofluorimeter (Horiba Jobin Yvon, Longjumeau, France) with double-grating excitation and emission monochromators for luminescence measurements over 400 to $700 \mathrm{~nm}$ wavelength range with a $0.1 \mathrm{~nm}$ step. PL spectra deconvolutions were carried out with OriginPro 8 SR4 (OriginLab Corp., Northampton, MA, USA) software 
using the Fit Multiple Peak procedure. The luminescence decay kinetics were studied by the excitation of a pulsed diode laser $(\lambda=377 \mathrm{~nm}, \Delta \tau=1.5 \mathrm{~ns})$ and a Xenon $450 \mathrm{~W}$ Ushio UXL-450S/O lamp (355 nm). Processing of the luminescence decay curves was carried out using the Fit Exponential procedure of an OriginPro 8 SR4 software. All of the decay curves were described by two exponentials (criterion Adj. R-Square $>0.998$ ). The final data were averaged over 5 measurements.

\section{Results and Discussion}

The general idea of high pure substances synthesis is that the best results could be obtained when we used the minimal set of initial preparations to conduct the synthesis reaction.

In our case the formal synthesis was described by the heterophase reaction (1)

$$
\mathrm{Al}_{2} \mathrm{O}_{3}^{\mathrm{s}}+38 \mathrm{Hq}^{\mathrm{v}} \rightarrow \mathrm{Alq}_{3}^{\mathrm{s}}+3 \mathrm{H}_{2} \mathrm{O}^{\mathrm{v}}
$$

The heterophase synthesis was conducted in a two-zone resistive furnace in a quartzglass reactor (Figure 3). The 8-Hq source was placed at $328-333 \mathrm{~K}\left(\mathrm{~T}_{1}\right)$ at the closed end of the reactor, while $\mathrm{Al}_{2} \mathrm{O}_{3}$ powder was placed in a hot zone at $463-513 \mathrm{~K}\left(\mathrm{~T}_{2}\right)$. To move the equilibrium towards the reaction products, we captured the water vapor by solid preliminary dried $\mathrm{P}_{2} \mathrm{O}_{5}$, which was placed in the quartz glass vessel at the cold open end of the reactor at temperature about $308-318 \mathrm{~K}\left(\mathrm{~T}_{3}\right)$. There was no need to put a pre-desiccant between $\mathrm{P}_{2} \mathrm{O}_{5}$, and the open end of the tube with its total length $20 \mathrm{~cm}$ because the flux of 8$\mathrm{Hq}$ and $\mathrm{H}_{2} \mathrm{O}$ vapors was directed from the closed end to the open end of the reactor. During the test experiments without $\mathrm{Al}_{2} \mathrm{O}_{3}$ preparation, we did not observe $\mathrm{P}_{2} \mathrm{O}_{5}$ degradation for $50 \mathrm{~h}$ of the processing.
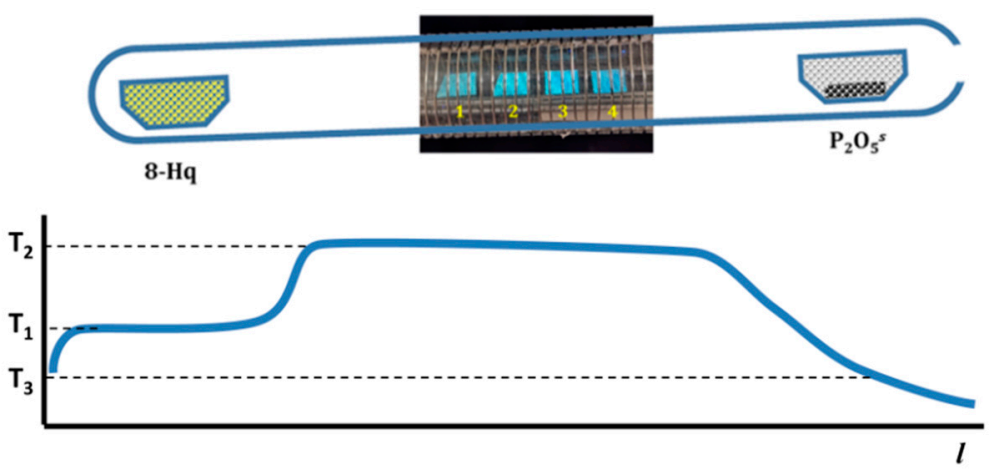

Figure 3. Scheme of setup for synthesis of $\mathrm{Alq}_{3}$ and temperature distribution in the setup.

Analysis of grains morphology after synthesis showed that the grain size distribution was the same as for the initial $\mathrm{Al}_{2} \mathrm{O}_{3}$ powder (Figure 4a). However, under UV lighting, we observed bright green-yellowish photoluminescence for the grains treated under 8-Hq vapor (Figure $4 \mathrm{~b}$ right half). SEM analysis in SE mode showed that the number of output secondary electrons form initial $\mathrm{Al}_{2} \mathrm{O}_{3}$ grains (Figure $4 \mathrm{c}$ left half) was more than that from the grains treated under 8 -Hq vapor (Figure $4 \mathrm{c}$ right half), because we observed a brighter image for the initial $\mathrm{Al}_{2} \mathrm{O}_{3}$ grains. All these observations indicated that we synthesized a new compound on $\mathrm{Al}_{2} \mathrm{O}_{3}$ grains surface and the thickness of the product was very small.

Spectral analysis showed that depending on a sample position in the furnace (see Figure 3 ) the $\lambda_{\mathrm{PL}}^{\max }$ shifted from $496 \mathrm{~nm}$ to $474 \mathrm{~nm}\left(\lambda^{\mathrm{exc}}=365 \mathrm{~nm}\right)$ (Figure 5) with the corresponding increase of PL intensity more than in seven times (Table 2). 


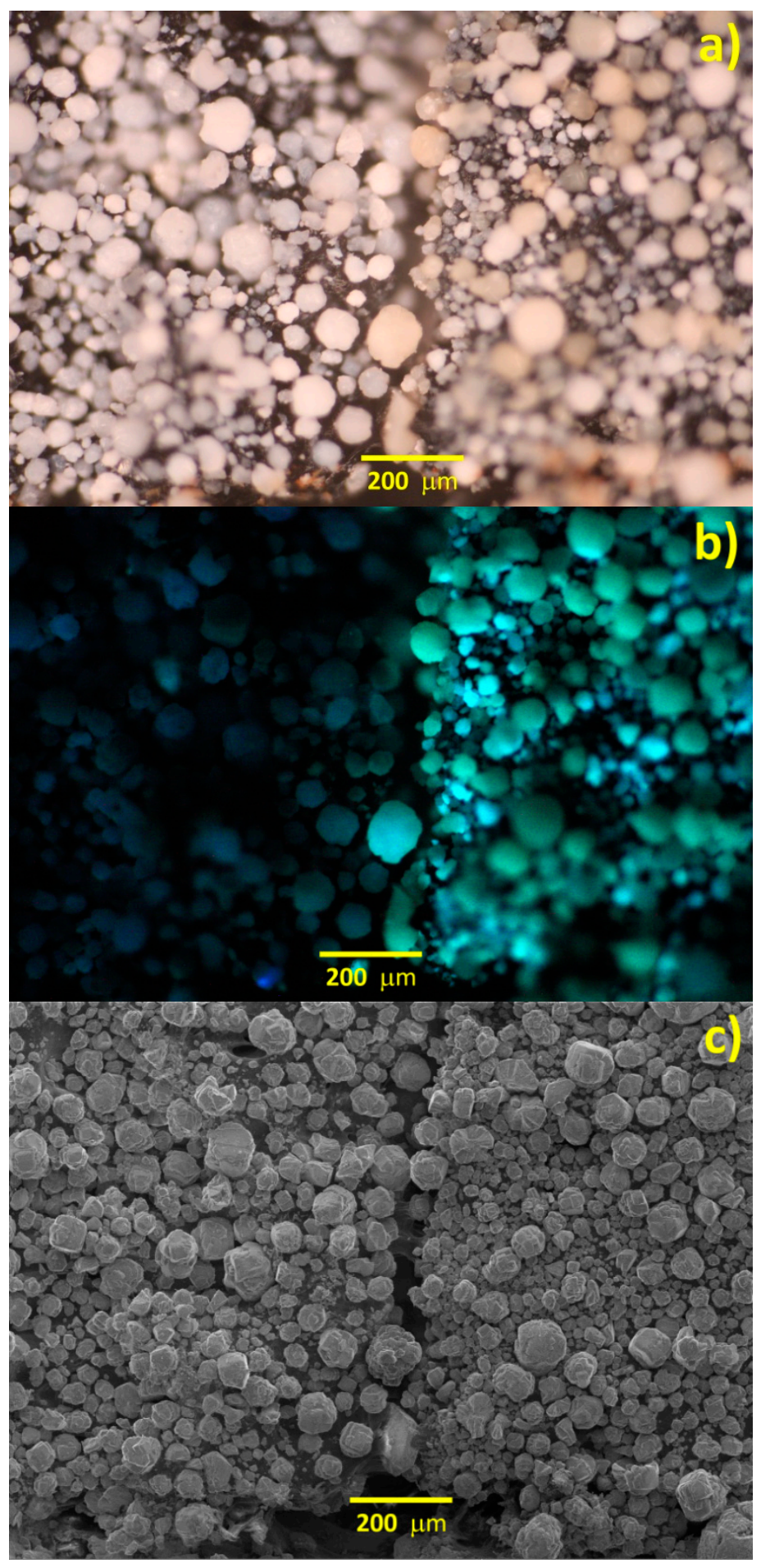

Figure 4. Microphotographs (a,b) and SEM image (c) of powder $\mathrm{Al}_{2} \mathrm{O}_{3}$ preparations under day light (a) and UV lighting (b) before (left half) and after heat treatment under 8-Hq vapor (right half). 


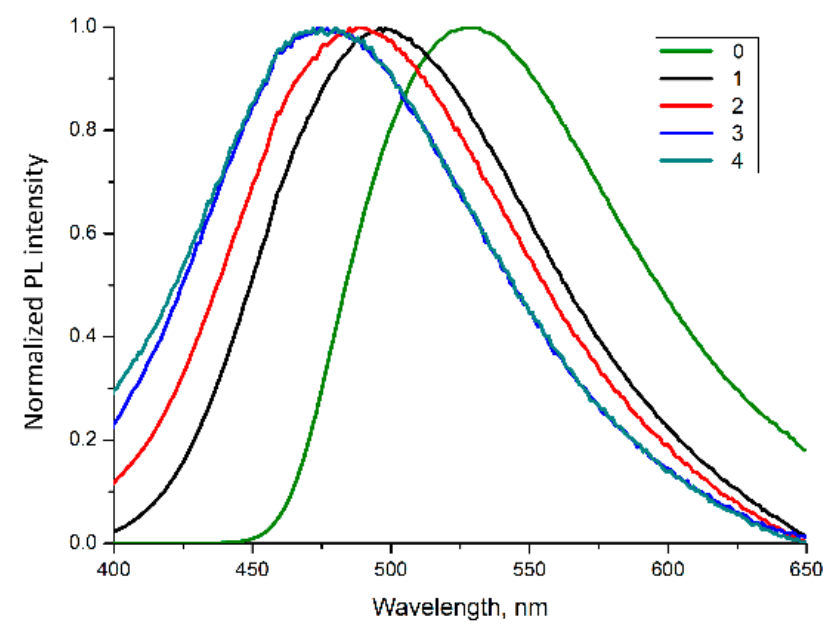

Figure 5. Normalized PL spectra $\left(\lambda^{\text {exc }}=365 \mathrm{~nm}\right.$ ) of $\mathrm{Alq}_{3}$ samples synthesized by the direct reaction at $\mathrm{T}=463 \mathrm{~K}$ and by wet synthesis. The numbers correspond to the position of samples in the furnace at the high-temperature synthesis (see Figure 1 and Table 2).

Table 2. PL peaks parameters for $\mathrm{Alq}_{3}$ samples synthesized by the direct synthesis and by the wet technique [20] (number 0).

\begin{tabular}{ccccc}
\hline Number & Peak Area & FWHM, nm & Center, nm & Height, cps \\
\hline 0 & $1.05 \times 10^{10}$ & 113.00 & 527 & $8.69 \times 10^{7}$ \\
1 & $8.43 \times 10^{8}$ & 113.94 & 496 & $7.04 \times 10^{6}$ \\
2 & $3.40 \times 10^{8}$ & 118.47 & 489 & $2.72 \times 10^{6}$ \\
3 & $2.03 \times 10^{8}$ & 119.47 & 480 & $1.61 \times 10^{6}$ \\
4 & $1.31 \times 10^{8}$ & 122.47 & 474 & $1.02 \times 10^{6}$ \\
\hline
\end{tabular}

Low PL intensity of direct-synthesized $\mathrm{Alq}_{3}$ preparations comparing to the wetsynthesized $\mathrm{Alq}_{3}$ we explained by very small thickness of the synthesized compound on the surface of $\mathrm{Al}_{2} \mathrm{O}_{3}$ grains. The hypsochromic shift of PL maximum for the direct-synthesized $\mathrm{Alq}_{3}$ comparing with the wet-synthesized $\mathrm{Alq}_{3}$ could results from summarizing of $\mathrm{PL}$ lighting with the reflected excitation light from the interface surface of $\mathrm{Alq}_{3}$ and $\mathrm{Al}_{2} \mathrm{O}_{3}$. We must also take into consideration the scheme of polymorph transformation for $\mathrm{Alq}_{3}$ [22]. In the case of $\delta-\mathrm{Alq}_{3}\left(\right.$ or $\gamma-\mathrm{Alq}_{3}$ ) the PL maximum in the films was found to be $474 \mathrm{~nm}$ [23].

Analysis of PL decay kinetics (Table 3, Figures S1-S5) showed that they were successfully described by two-exponential equation. The short-lived centers had the lifetime about $2 \mathrm{~ns}$, while the long-lived centers had the lifetime of 16-17 ns. We observed that the wet-synthesized sample has a decay kinetics specific for $\alpha$-Alq ${ }_{3}$ [20], and it was longer than that for the samples obtained by the direct synthesis.

Table 3. PL decay kinetics of $\mathrm{Alq}_{3}$ samples synthesized by the direct synthesis and by the wet technique [20] (number 0 ), described by the equation $\mathrm{Y}=\mathrm{A} 1 \times \exp (-\mathrm{x} / \tau 1)+\mathrm{A} 2 \times \exp (-\mathrm{x} / \tau 2)+\mathrm{Y} 0$.

\begin{tabular}{ccccccc}
\hline Number & $\begin{array}{c}\lambda_{\text {PL }}^{\max } \\
\text { nm }\end{array}$ & Y0 & A1 & $\begin{array}{c}\boldsymbol{\tau 1} \\
\text { ns }\end{array}$ & A2 & $\begin{array}{c}\boldsymbol{\tau} \text { 2, } \\
\text { ns }\end{array}$ \\
\hline 0 & 527 & $102.39 \pm 0.71$ & $6503 \pm 134$ & $8.56 \pm 0.19$ & $8945 \pm 172$ & $21.14 \pm 0.15$ \\
1 & 496 & $127.54 \pm 1.01$ & $41694 \pm 822$ & $2.78 \pm 0.03$ & $5694 \pm 58$ & $16.91 \pm 0.11$ \\
2 & 489 & $80.30 \pm 0.92$ & $39579 \pm 800$ & $2.71 \pm 0.03$ & $6413 \pm 50$ & $17.23 \pm 0.09$ \\
3 & 480 & $45.02 \pm 0.87$ & $51000 \pm 1319$ & $2.31 \pm 0.03$ & $6596 \pm 44$ & $16.67 \pm 0.08$ \\
4 & 474 & $30.73 \pm 0.86$ & $78664 \pm 2359$ & $1.97 \pm 0.02$ & $6395 \pm 40$ & $16.17 \pm 0.07$ \\
\hline
\end{tabular}

We failed to find any data on PL decay kinetics for different polymorphs of $\mathrm{Alq}_{3}$ in the literature. Therefore, we assumed that for $\delta-\mathrm{Alq}_{3}\left(\right.$ or $\gamma$ - $\left.\mathrm{Alq}_{3}\right)$, which were probably obtained in our experiments, the PL decay kinetics was shorter than for $\alpha-\mathrm{Alq}_{3}$. 
According to ICP-MS analysis, as-synthesized $\mathrm{Alq}_{3}$ has the chemical purity of $99.998 \mathrm{wt} \%$ (Figure 6). The major impurities were $\mathrm{Si}$ and $\mathrm{K}$. We assume these impurities are inherited from the container material: a quartz-glass reactor and a quartz-glass vessel with 8-Hq.

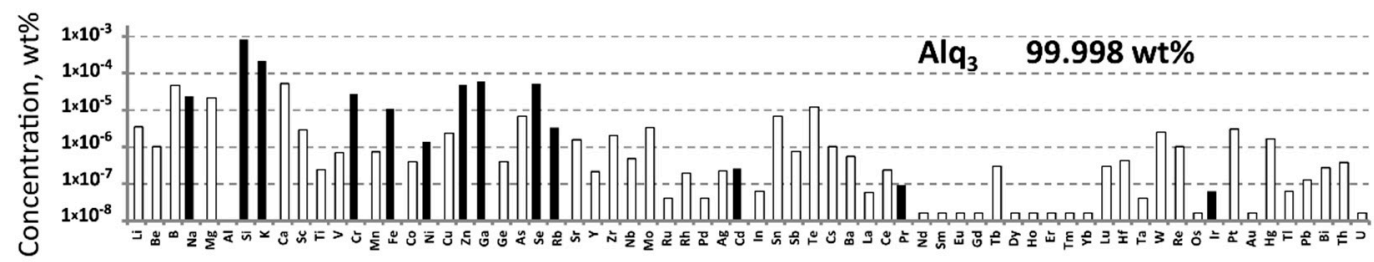

Figure 6. Impurity concentrations determined by ICP-MS in as-synthesized $\mathrm{Alq}_{3}$.

High purity aluminum oxide is often termed as high purity alumina (HPA). It is a highvalue, white, granular chemical produced commercially. Analysis of the world alumina market showed that $5 \mathrm{~N}$ and $6 \mathrm{~N} \mathrm{Al}_{2} \mathrm{O}_{3}$ are available preparation at a comparatively low price [24]. 8-Hq is simply purified by a sublimation procedure to the level of $99.999 \mathrm{wt} \%$. Thus, we could say that there are commercially available sources for simple synthesis of high pure $\mathrm{Alq}_{3}$.

\section{Conclusions}

A new approach to the synthesis of tris(8-hydroxyquinolate) aluminum showed the fundamental possibility of preparation an electroluminescent high-purity material using fairly simple operations and an easy procedure. One of the advantages of the produced material is its stability to the environment. We did not observe any degradation when storage the synthesized preparations in common used vessels without additional sealing or filling with an inert gas. Further refinement of the developed technique, for instance, using glassy carbon reactor, will make it possible to obtain cheap and even more high-pure materials for OLED technologies.

Supplementary Materials: The following supporting information can be downloaded at: https: / / www.mdpi.com/article/10.3390/ma15030734/s1, Figure S1: PL decay kinetics at $527 \mathrm{~nm}$ for the Alq3 preparation obtained by the «wet» synthesis, Figure S2: PL decay kinetics at $496 \mathrm{~nm}$ for the Alq3 preparation (N1) obtained by the direct synthesis, Figure S3: PL decay kinetics at $489 \mathrm{~nm}$ for the Alq3 preparation (N2) obtained by the direct synthesis, Figure S4: PL decay kinetics at $480 \mathrm{~nm}$ for the Alq3 preparation (N3) obtained by the direct synthesis, Figure S5: PL decay kinetics at $474 \mathrm{~nm}$ for the Alq3 preparation (N4) obtained by the direct synthesis.

Author Contributions: Conceptualization, R.A. and K.K.; data curation, A.K.; formal analysis, A.B. and I.A.; funding acquisition, R.A.; investigation, K.K., A.B., M.Z. and A.P.; methodology, A.K.; project administration, K.K.; resources, M.Z.; supervision, M.Z.; validation, A.P.; visualization, A.B.; writing-original draft, R.A. and A.B.; writing—review and editing, I.A. All authors have read and agreed to the published version of the manuscript.

Funding: The research was financially supported by the Ministry of Science and Higher Education of Russia by the project FSSM-2020-0005.

Conflicts of Interest: The authors declare no conflict of interest.

\section{References}

1. So, F. Organic Electronics: Materials, Processing, Devices and Applications; CRC Press: Boca Raton, FL, USA, 2010.

2. Irimia-Vladu, M.; Glowacki, E.D.; Sariciftci, N.S.; Bauer, S. Green Materials for Electronics; Wiley-VCH: Weinheim, Germany, 2018; ISBN 978-3-527-33865-8.

3. Wu, X.; Ma, Y.; Zhang, G.; Chu, Y.; Du, J.; Zhang, Y.; Li, Z.; Duan, Y.; Fan, Z.; Huang, J. Thermally Stable, Biocompatible, and Flexible Organic Field-Effect Transistors and Their Application in Temperature Sensing Arrays for Artificial Skin. Adv. Funct. Mater. 2015, 25, 2138-2146. [CrossRef]

4. Stadlober, B.; Zirkl, M.; Irimia-Vladu, M. Route towards Sustainable Smart Sensors: Ferroelectric Polyvinylidene Fluoride-Based Materials and Their Integration in Flexible Electronics. Chem. Soc. Rev. 2019, 48, 1787-1825. [CrossRef] [PubMed] 
5. Nalwa, H.S. Handbook of Organic Electronics and Photonics; American Scientific Pub.: Stevenson Ranch, CA, USA, 2008.

6. Anthony, J.E.; Arias, A.C.; Bergsmann, M.; Blanchet, G.; Cantatore, E.; Halik, M.; Heuken, M.; Horowitz, G.; Huang, J.; Katz, H.E.; et al. Organic Electronics; Klauk, H., Ed.; Wiley-VCH: Weinheim, Germany, 2006. [CrossRef]

7. Peyghambarian, N.; Fallahi, M.; Piprek, J.; Sun, S.; Prigodin, V.N.; Epstein, A.J.; Meng, X.; Zhu, W.; Tian, H.; Li, Y.; et al. Introduction to Organic Electronic and Optoelectronic Materials and Devices, 2nd ed.; CRC Press: New York, NY, USA, 2016. [CrossRef]

8. Wong, W.S.; Salleo, A. Flexible Electronics: Materials and Applications, 1st ed.; Springer Publishing Company, Incorporated: New York, NY, USA, 2009.

9. Shinar, R.; Shinar, J. Organic Electronics in Sensors and Biotechnology; McGraw-Hill: New York, NY, USA, 2009.

10. Bao, Z.; Locklin, J. Organic Field-Effect Transistors; Bao, Z., Locklin, J., Eds.; CRC Press: New York, NY, USA, 2018. [CrossRef]

11. Martins, J.; Sousa, L. Bioelectronic Vision; World Scientific Publishing Co. Pte. Ltd., Toh Tuck Link: Singapore, 2009; ISBN 978-981-279-430-7.

12. Brabec, C.J.; Dyakonov, V.; Parisi, J.; Sariciftci, N.S. Organic Photovoltaics: Concepts and Realization; Springer Series in Materials Science: Berlin/Heidelberg, Germany, 2013.

13. Perlin, J.; Hepp, A.F.; Bailey, S.G.; Raffaelle, R.P.; Blankenship, R.R.; Lane, P.A.; Kafafi, Z.H.; Persson, N.; Inganas, O.; Gregg, B.A.; et al. Organic Photovoltaics; Sam-Shajing, S., Niyazi Serdar, S., Eds.; CRC Press: New York, NY, USA, 2017. [CrossRef]

14. Brutting, W. Physics of Organic Semiconductors; WILEY-VCH Verlag GmbH \& Co. KGaA: Weinheim, Germany, 2005; ISBN 978-3-527-40550-3.

15. Tang, C.W.; VanSlyke, S.A. Organic Electroluminescent Diodes. Appl. Phys. Lett. 1987, 51, 913-915. [CrossRef]

16. Electronic Grade Gallium Arsenide. Available online: https://chem.libretexts.org/@go/page/212888 (accessed on 20 November 2021).

17. Maurits, J.E.A. Silicon Production. In Treatise on Process Metallurgy; Elsevier Ltd.: Vancouver, WA, USA, 2014; pp. 919-948. [CrossRef]

18. Available online: https://www.americanelements.com/tris-8-hydroxyquinoline-aluminum-2085-33-8 (accessed on 25 November 2021).

19. Available online: https://cymitquimica.com/products/TR-T875045/2085-33-8/tris8-hydroxychinolinaluminum/ (accessed on 25 November 2021).

20. Avetissov, I.C.; Akkuzina, A.A.; Avetisov, R.I.; Khomyakov, A.V.; Saifutyarov, R.R. Non-Stoichiometry of Tris(8-Hydroxyquinoline) Aluminium: Is It Possible? CrystEngComm 2016, 18, 2182-2188. [CrossRef]

21. Pruszkowski, E.; Life, P.E. Total quant analysis of teas and wines by ICP-MS. In Perkin Elmer Life and Analytical Sciences; Field Application Report; ICP Mass Spectrometry: New York, NY, USA, 2004.

22. Avetisov, R.I.; Akkuzina, A.A.; Cherednichenko, A.G.; Khomyakov, A.V.; Avetissov, I.C. Polymorphism of Tris(8-Hydroxyquinoline)aluminu Gallium, and Indium. Dokl. Chem. 2014, 454, 6-8. [CrossRef]

23. Muccini, M.; Loi, M.A.; Kenevey, K.; Zamboni, R.; Masciocchi, N.; Sironi, A. Blue Luminescence of Facial Tris(Quinolin-8Olato)Aluminum(III) in Solution, Crystals, and Thin Films. Adv. Mater. 2004, 16, 861-864. [CrossRef]

24. Available online: https://www.persistencemarketresearch.com/market-research/high-purity-alumina-market/toc (accessed on 25 November 2021). 